\title{
The Sub-Supersolution Method and Extremal Solutions of Quasilinear Elliptic Equations in Orlicz-Sobolev Spaces
}

\author{
Ge Dong $\mathbb{D}^{1}$ and Xiaochun Fang $\mathbb{D}^{2}$ \\ ${ }^{1}$ College of Information on Technology, Shanghai Jian Qiao University, Shanghai 201306, China \\ ${ }^{2}$ Department of Mathematics, Tongji University, Shanghai 200092, China \\ Correspondence should be addressed to Xiaochun Fang; xfang@tongji.edu.cn
}

Received 13 June 2018; Accepted 24 July 2018; Published 5 August 2018

Academic Editor: Alberto Fiorenza

Copyright (c) 2018 Ge Dong and Xiaochun Fang. This is an open access article distributed under the Creative Commons Attribution License, which permits unrestricted use, distribution, and reproduction in any medium, provided the original work is properly cited.

We prove the existence of extremal solutions of the following quasilinear elliptic problem $-\sum_{i=1}^{N}\left(\partial / \partial x_{i}\right) a_{i}(x, u(x), D u(x))+$ $g(x, u(x), D u(x))=0$ under Dirichlet boundary condition in Orlicz-Sobolev spaces $W_{0}^{1} L_{M}(\Omega)$ and give the enclosure of solutions. The differential part is driven by a Leray-Lions operator in Orlicz-Sobolev spaces, while the nonlinear term $g: \Omega \times \mathbb{R} \times \mathbb{R}^{N} \longrightarrow \mathbb{R}$ is a Carathéodory function satisfying a growth condition. Our approach relies on the method of linear functional analysis theory and the sub-supersolution method.

\section{Introduction}

The aim of this paper is to study some qualitative properties of solutions of the following quasilinear elliptic problem:

$$
-\sum_{i=1}^{N} \frac{\partial}{\partial x_{i}} a_{i}(x, u(x), D u(x))+g(x, u(x), D u(x))=0
$$

$$
\begin{gathered}
\text { in } \Omega \\
u=0 \\
\text { on } \partial \Omega,
\end{gathered}
$$

on a bounded domain $\Omega \subset \mathbb{R}^{N}$ with a Lipschitz boundary $\partial \Omega$ in Orlicz-Sobolev spaces. The differential part is driven by a Leray-Lions operator, while the nonlinear term $g: \Omega \times$ $\mathbb{R} \times \mathbb{R}^{N} \longrightarrow \mathbb{R}$ is a Carathéodory function satisfying a growth condition.

In [1, Chapter 3], the differential part of (1) is a LerayLions operator in Sobolev spaces and the nonlinearity $g(x, s, \xi)$ satisfies the growth condition:

$$
|g(x, s, \xi)| \leq k_{1}(x)+c|\xi|^{p-1},
$$

with the constant $c>0$ and $k_{1}(x) \in L_{+}^{p^{\prime}}(\Omega)$, for a.e. $x \in \Omega$, all $s \in \mathbb{R}$ and all $\xi \in \mathbb{R}^{N}$, where $p^{\prime}$ is the conjugate Hölder exponent to $p$, i.e., $1 / p+1 / p^{\prime}=1$. In [2], the nonlinearity $g(x, s, \xi)$ satisfies the growth condition:

$$
|g(x, s, \xi)| \leq k_{1}(x)+c|\xi|^{r},
$$

with the constant $c \geq 0$ and $k_{1}(x) \in L^{q}(\Omega), k_{1} \geq 0, q>\left(p^{*}\right)^{\prime}$, $0 \leq r \leq p /\left(p^{*}\right)^{\prime}$, for a.e. $x \in \Omega$, all $s \in \mathbb{R}$ and all $\xi \in \mathbb{R}^{N}$, where $p^{*}$ is the Sobolev conjugate of $p$. Faria [2] pointed that the condition (3) is more general than (2) because $p-1<$ $p /\left(p^{*}\right)^{\prime}$. However, $p /\left(p^{*}\right)^{\prime}=p-1-p / N<p-1$ if $0<p<N$. Hence, the growth condition (3) is not more general than (2).

When trying to weaken the restriction on the Leray-Lions operator and the growth condition (2), one is led to replace $W_{0}^{1, p}(\Omega)$ with $W_{0}^{1} L_{M}(\Omega)$ built from an Orlicz space $L_{M}(\Omega)$ instead of $L^{p}(\Omega)$, where $M$ is an $N$-function. The choice $M(t)=|t|^{p}, p>1$ leads to [1, Theorem 3.17]. A nonstandard example is $M(t)=\int_{0}^{|u|} \log \left(1+|t|^{q}\right)|t|^{p-2} t d t$ (see, e.g., $[2,3]$ ).

Many papers used the surjectivity result for pseudomonotone operators (see, e.g., [1, Theorem 2.99]) defined on reflexive spaces to prove the existence of the solution (see, e.g., $[1,2,4,5])$. Our method does not need the reflexivity of the spaces. It is well known that the Orlicz space is reflex if and only if both $M$ and its complementary function $\bar{M}$ satisfy $\Delta_{2}$-condition. However, there exist many spaces without reflexivity. For example, let $M(u)=(1+|u|) \ln (1+|u|)-$ 
$|u|$; then $M$ satisfies $\Delta_{2}$-condition, but its complementary function $\bar{M}(v)=\exp (|v|)-|v|-1$ does not satisfy $\Delta_{2}{ }^{-}$ condition; i.e., $L_{M}(\Omega)$ is not reflexive.

In this paper, we get rid of the restriction of the reflexivity of the spaces and get a weak solution for (1) in Orlicz-Sobolev spaces by using a linear functional analysis method. We also give the enclosure of solutions and prove the existence of extremal solutions.

This paper is organized as follows. Section 2 contains some preliminaries and some technical lemmas which will be needed. In Section 3, we use the linear functional analysis method to prove the existence of solutions for (1) in separable Orlicz-Sobolev spaces and the sub-supersolutions method to give the enclosure of solutions and the existence of extremal solutions between a subsolution and a supersolution. We also get the compactness and directness of the solutions set.

For some results, we also refer to [6-13].

\section{Preliminaries}

For quick reference, we recall some basic results of Orlicz spaces. Good references are Adams [14, Chapter 8], Krasnosel'skil [15], Chen [16], and Gossez [17].

2.1. N-Function. Let $M: \mathbb{R}^{+} \longrightarrow \mathbb{R}^{+}$be an $N$-function; i.e., $M$ is continuous, convex, with $M(u)>0$ for $u>0$, $M(u) / u \longrightarrow 0$ as $u \longrightarrow 0$, and $M(u) / u \longrightarrow+\infty$ as $u \longrightarrow+\infty$. Equivalently, $M$ admits the representation $M(u)=\int_{0}^{u} \phi(t) d t$, where $\phi: \mathbb{R}^{+} \longrightarrow \mathbb{R}^{+}$is a nondecreasing, right continuous function, with $\phi(0)=0, \phi(t)>0$ for $t>0$, and $\phi(t) \longrightarrow+\infty$ as $t \longrightarrow+\infty$.

The $N$-function $\bar{M}$ conjugated to $M$ is defined by $\bar{M}(v)=$ $\int_{0}^{v} \psi(s) d s$, where $\psi: \mathbb{R}^{+} \longrightarrow \mathbb{R}^{+}$is given by $\psi(s)=\sup \{t$ : $\phi(t) \leq s\}$.

$\phi, \psi$ are called the right-hand derivatives of $M, \bar{M}$, respectively.

The $N$-function $M$ is said to satisfy the $\Delta_{2}$ condition near infinity ( $M \in \Delta_{2}$, for short), if, for some $k>1$ and $\tilde{u}>0$, $M(2 u) \leq k M(u), \forall u \geq \widetilde{u}$.

Moreover, one has the following Young inequality: $u v \leq$ $M(u)+\bar{M}(v), \forall u, v \geq 0$.

For the $N$-function $M$ one defines the Sobolev conjugate $M_{*}$ by $M_{*}^{-1}(t)=\int_{0}^{t}\left(M^{-1}(\tau) / \tau^{(N+1) / N}\right) d \tau, t \geq 0$.

Let $P, Q$ be two $N$-functions, we say that $P$ grows essentially less rapidly than $Q$ near infinity, denoted as $P \ll$ $Q$, if for every $\varepsilon>0, P(t) / Q(\varepsilon t) \longrightarrow 0$ as $t \longrightarrow+\infty$. This is the case if and only if $\lim _{t \rightarrow+\infty} Q^{-1}(t) / P^{-1}(t)=0$.

We will extend these $N$-functions into even functions on all $\mathbb{R}$.

For a measurable function $u$ on $\Omega$, its modular is defined by $\rho_{M}(u)=\int_{\Omega} M(|u(x)|) d x$.

2.2. Orlicz Spaces. Let $\Omega$ be an open and bounded subset of $\mathbb{R}^{N}$ and $M$ be an $N$-function. The Orlicz class $\mathscr{K}_{M}(\Omega)$ (resp., the Orlicz space $L_{M}(\Omega)$ ) is defined as the set of (equivalence classes of) real valued measurable functions $u$ on $\Omega$ such that $\rho_{M}(u)<+\infty\left(\right.$ resp. $\rho_{M}(u / \lambda)<+\infty$ for some $\left.\lambda>0\right) . L_{M}(\Omega)$ is a Banach space under the (Luxemburg) norm:

$$
\|u\|_{(M)}=\inf \left\{\lambda>0: \rho_{M}\left(\frac{u}{\lambda}\right) \leq 1\right\},
$$

and $\mathscr{K}_{M}(\Omega)$ is a convex subset of $L_{M}(\Omega)$ but not necessarily a linear space. The closure in $L_{M}(\Omega)$ of the set of bounded measurable functions with compact support in $\bar{\Omega}$ is denoted by $E_{M}(\Omega)$.

The equality $E_{M}(\Omega)=L_{M}(\Omega)$ holds if and only if $M \epsilon$ $\Delta_{2}$; moreover, $L_{M}(\Omega)$ is separable.

$L_{M}(\Omega)$ is reflexive if and only if $M \in \Delta_{2}$ and $\bar{M} \in \Delta_{2}$.

Convergences in norm and in modular are equivalent if and only if $M \in \Delta_{2}$.

The dual space of $E_{M}(\Omega)$ can be identified with $L_{\bar{M}}(\Omega)$ by means of the pairing $\int_{\Omega} u(x) v(x) d x$, and the dual norm of $L_{\bar{M}}(\Omega)$ is equivalent to $\|\cdot\|_{(\bar{M})}$.

2.3. Orlicz-Sobolev Spaces. We now turn to the OrliczSobolev space: $W^{1} L_{M}(\Omega)$ (resp., $W^{1} E_{M}(\Omega)$ ) is the space of all functions $u$ such that $u$ and its distributional partial derivatives lie in $L_{M}(\Omega)$ (resp., $E_{M}(\Omega)$ ). It is a Banach spaces under the norm

$$
\|u\|_{\Omega, M}=\sum_{|\alpha| \leq 1}\left\|D^{\alpha} u\right\|_{(M)} .
$$

Denote $\|D u\|_{(M)}=\||D u|\|_{(M)}$ and $\|u\|_{1, M}=\|u\|_{(M)}+\|D u\|_{(M)}$. Clearly, $\|u\|_{1, M}$ is equivalent to $\|u\|_{\Omega, M}$.

Thus $W^{1} L_{M}(\Omega)$ and $W^{1} E_{M}(\Omega)$ can be identified with subspaces of the product of $N+1$ copies of $L_{M}(\Omega)$. Denoting this product by $\Pi L_{M}$, we will use the weak topologies $\sigma\left(\Pi L_{M}, \Pi E_{\bar{M}}\right)$ and $\sigma\left(\Pi L_{M}, \Pi L_{\bar{M}}\right)$.

If $M \in \Delta_{2}$, then $W^{1} L_{M}(\Omega)=W^{1} E_{M}(\Omega)$. If $M \in \Delta_{2}$ and $\bar{M} \in \Delta_{2}$, then $W^{1} L_{M}(\Omega)=W^{1} E_{M}(\Omega)$ are reflexive; thus the weak topologies $\sigma\left(\Pi L_{M}, \Pi E_{\bar{M}}\right)$ and $\sigma\left(\Pi L_{M}, \Pi L_{\bar{M}}\right)$ are equivalent.

Lemma 1 (See [18, Lemma 2.2]). For all $u \in W_{0}^{1} L_{M}(\Omega)$, one has

$$
\int_{\Omega} M\left(\frac{|u(x)|}{\operatorname{diam} \Omega}\right) d x \leq \int_{\Omega} M(|D u(x)|) d x,
$$

where diam $\Omega$ is the diameter of $\Omega$.

Lemma 2 (See [19, Lemma 1]). Let meas $\Omega$ be bounded and $\varphi: \mathbb{R}^{+} \longrightarrow \mathbb{R}^{+}, \varphi(0)=0, \varphi(r) \longrightarrow+\infty$ for $r \longrightarrow+\infty$. Then

$$
\begin{aligned}
\frac{\int_{\Omega} \varphi(|D u(x)|)|D u(x)| d x}{\int_{\Omega}|D u(x)| d x} & \longrightarrow+\infty \\
& \text { if } \int_{\Omega}|D u(x)| d x \longrightarrow+\infty .
\end{aligned}
$$

Lemma 3 (See [20, Lemma 2.1]). If $u \in W^{1} L_{M}(\Omega)$, then $u^{+}$, $u^{-} \in W^{1} L_{M}(\Omega)$ and

$$
\begin{aligned}
& D u^{+}= \begin{cases}D u, & \text { if } u>0 \\
0, & \text { if } u \leq 0\end{cases} \\
& D u^{-}= \begin{cases}0, & \text { if } u \geq 0 \\
-D u, & \text { if } u<0\end{cases}
\end{aligned}
$$

Here $u^{+}=\max \{u, 0\}, u^{-}=-\min \{u, 0\}$. 


\section{Main Results}

Let $\Omega$ be a bounded domain in $\mathbb{R}^{N}(N \geq 1)$ with Lipschitz boundary, $M, P$ be two $N$-functions, and $\bar{M}, \bar{P}$ be the complementary functions of $M, P$, respectively. Assume that $M$ satisfies the $\Delta_{2}$ condition near infinity and $P \ll M$. By Theorem 2.2 and Proposition 2.1 in [21] the embeddings $W_{0}^{1} L_{M}(\Omega) \hookrightarrow$ $L_{P}(\Omega)$ and $W_{0}^{1} L_{M}(\Omega) \hookrightarrow L_{M}(\Omega)$ are compact.

Let $A$ be the following quasilinear elliptic differential operator in divergence form:

$$
A u(x)=-\sum_{i=1}^{N} \frac{\partial}{\partial x_{i}} a_{i}(x, u(x), D u(x))
$$

where the coefficients $a_{i}: \Omega \times \mathbb{R} \times \mathbb{R}^{N} \longrightarrow \mathbb{R}, i=1, \ldots, N$, are assumed to satisfy the following:

(H1) Each function $a_{i}(x, s, \xi)$ is a Carathéodory function. Also there exists a positive constant $\beta$ and a nonnegative function $k_{0} \in E_{\bar{M}}(\Omega)$ such that

$$
\begin{aligned}
& \left|a_{i}(x, s, \xi)\right| \\
& \quad \leq \beta\left[k_{0}(x)+\bar{P}^{-1}(M(|s|))+\bar{M}^{-1}(M(|\xi|))\right]
\end{aligned}
$$

for a.e. $x \in \Omega$ and for all $s \in \mathbb{R}, \xi \in \mathbb{R}^{N}$.

(H2) $\sum_{i=1}^{N}\left(a_{i}(x, s, \xi)-a_{i}\left(x, s, \xi^{\prime}\right)\right)\left(\xi_{i}-\xi_{i}^{\prime}\right)>0$ for a.e. $x \in \Omega$, all $s \in \mathbb{R}$, and all $\xi, \xi^{\prime} \in \mathbb{R}^{N}$ with $\xi \neq \xi^{\prime}$.

(H3) $\sum_{i=1}^{N} a_{i}(x, s, \xi) \xi_{i} \geq v M(|\xi|)-k(x)$ for a.e. $x \in \Omega$, all $s \in \mathbb{R}$, and all $\xi \in \mathbb{R}^{N}$, with some constant $v>0$ and a function $k \in L^{1}(\Omega)$.

The differential operator $A$ can be seen as a mapping from $W_{0}^{1} L_{M}(\Omega)$ into its dual space $\left(W_{0}^{1} L_{M}(\Omega)\right)^{*}$ given by

$$
\langle A u, v\rangle=\sum_{i=1}^{N} \int_{\Omega} a_{i}(x, u(x), D u(x)) \frac{\partial v(x)}{\partial x_{i}} d x
$$

for all $u, v \in W_{0}^{1} L_{M}(\Omega)$.

Example 4. (1) The p-Laplacian operator $\Delta_{p}=$ $\operatorname{div}\left(|D u|^{p-2} D u\right)$ is form $A$ with the coefficients $a_{i}$, $i=1, \ldots, N$, given by $a_{i}(x, s, \xi)=|\xi|^{p-2} \xi_{i}$ (see, e.g., $[1$, Example 2.110]).

(2) Let $p(t)$ be a given positive and continuous function which increases from 0 to $+\infty$ and $a_{i}(x, s, \xi)=(p(|\xi|) /|\xi|) \xi_{i}$. Then $a_{i}, i=1, \ldots, N$, satisfy the conditions (H1)-(H3).

Consider the following nonlinear elliptic equation:

$$
\begin{array}{rlrl}
A u+g(x, u, D u) & =0 & & \text { in } \Omega \\
u=0 & & \text { on } \partial \Omega .
\end{array}
$$

Here, $g: \Omega \times \mathbb{R} \times \mathbb{R}^{N} \longrightarrow \mathbb{R}$ is assumed to be a Carathéodory function.

Let $G$ denote the Nemytskij operator related to $g$ by

$$
G(u)(x)=g(x, u(x), D u(x)), \quad x \in \Omega .
$$

For $u, v \in L^{0}(\Omega)$, we use the standard notations: $u \wedge v=$ $\min \{u, v\}, u \vee v=\max \{u, v\}, u^{+}:=u \vee 0, u^{-}:=-u \wedge 0$, $u \leq v \Longleftrightarrow u(x) \leq v(x)$ for a.e. $x \in \Omega$. A weak solution of (12) is called a solution for short.

By Lemma 3, $W^{1} L_{M}(\Omega)$ is closed under $\vee$ and $\wedge$. In fact, since $u \vee v=v+(u-v)^{+}$and $u \wedge v=v-(u-v)^{-}, u \vee v$, $u \wedge v \in W^{1} L_{M}(\Omega)$, for any $u, v \in W^{1} L_{M}(\Omega)$.

The following lemma can be found in [5, Remark 3.1] as the setting of Musielak-Orlicz spaces. However, we give another proof.

Lemma 5. (a) $W^{1} L_{M}(\Omega)$ (resp., $W_{0}^{1} L_{M}(\Omega)$ ) is closed under " $\vee$ " and " $\wedge$ ", i.e., if $u, v \in W^{1} L_{M}(\Omega)\left(\right.$ resp., $\left.W_{0}^{1} L_{M}(\Omega)\right)$, then $u \vee v, u \wedge v \in W^{1} L_{M}(\Omega)$ (resp., $W_{0}^{1} L_{M}(\Omega)$ ).

(b) The mappings $\vee$ and $\wedge: W^{1} L_{M}(\Omega) \times W^{1} L_{M}(\Omega) \longrightarrow$ $W^{1} L_{M}(\Omega)$ (resp., $W_{0}^{1} L_{M}(\Omega) \times W_{0}^{1} L_{M}(\Omega) \longrightarrow W_{0}^{1} L_{M}(\Omega)$ ) are continuous, i.e., for any sequences $\left\{u_{n}\right\},\left\{v_{n}\right\}$ in $W^{1} L_{M}(\Omega)$ (resp., $W_{0}^{1} L_{M}(\Omega)$ ), if $u_{n} \longrightarrow u, v_{n} \longrightarrow v$ in $W^{1} L_{M}(\Omega)$ (resp., $W_{0}^{1} L_{M}(\Omega)$ ), then $u_{n} \vee v_{n} \longrightarrow u \vee v, u_{n} \wedge v_{n} \longrightarrow u \wedge v$ in $W^{1} L_{M}(\Omega)$ (resp., $W_{0}^{1} L_{M}(\Omega)$ ), as $n \longrightarrow \infty$.

Proof. (a) By Lemma 3, $W^{1} L_{M}(\Omega)$ (resp., $W_{0}^{1} L_{M}(\Omega)$ ) is closed under $\vee$ and $\wedge$. In fact, since $u \vee v=v+(u-v)^{+}$ and $u \wedge v=v-(u-v)^{-}, u \vee v, u \wedge v \in W^{1} L_{M}(\Omega)$ (resp., $W_{0}^{1} L_{M}(\Omega)$ ), for any $u, v \in W^{1} L_{M}(\Omega)\left(\right.$ resp., $W_{0}^{1} L_{M}(\Omega)$ ).

(b) Let $u_{n} \longrightarrow u, v_{n} \longrightarrow v$ in $W^{1} L_{M}(\Omega)$ (resp., $\left.W_{0}^{1} L_{M}(\Omega)\right)$, as $n \longrightarrow \infty$. Suppose that there exists $\varepsilon_{1}>0$ such that

$$
\left\|u_{n} \vee v_{n}-u \vee v\right\|_{(M)}>\varepsilon_{1}>0
$$

for any $n \in \mathbb{N}$, then $\rho_{M}\left(\left(u_{n} \vee v_{n}-u \vee v\right) / \varepsilon_{1}\right)>1$.

Therefore, we have $\int_{\Omega} M\left(\left(8 / \varepsilon_{1}\right)\left|u_{n}(x)-u(x)\right|\right) d x \longrightarrow 0$, and $\int_{\Omega} M\left(\left(8 / \varepsilon_{1}\right)\left|v_{n}(x)-v(x)\right|\right) d x \longrightarrow 0$, as $n \longrightarrow \infty$. By passing to a subsequence if necessary, $u_{n} \longrightarrow u, v_{n} \longrightarrow v$, a.e. in $\Omega$, as $n \longrightarrow \infty$, and there exist $f_{1}, f_{2} \in L^{1}(\Omega)$ such that $M\left(\left(8 / \varepsilon_{1}\right)\left|u_{n}(x)-u(x)\right|\right) \leq f_{1}(x)$, and $M\left(\left(8 / \varepsilon_{1}\right) \mid v_{n}(x)-\right.$ $v(x) \mid) \leq f_{2}(x)$, which yields that $M\left(\left(4 / \varepsilon_{1}\right)\left|u_{n}(x)\right|\right) \leq$ $(1 / 2) f_{1}+(1 / 2) M\left(\left(8 / \varepsilon_{1}\right)|u(x)|\right), M\left(\left(4 / \varepsilon_{1}\right)\left|v_{n}(x)\right|\right) \leq(1 / 2) f_{2}+$ $(1 / 2) M\left(\left(8 / \varepsilon_{1}\right)|v(x)|\right)$, for a.e. $x \in \Omega$.

Hence, $u_{n} \vee v_{n} \longrightarrow u \vee v$ a.e. in $\Omega$, as $n \longrightarrow \infty$, and

$$
\begin{aligned}
M & \left(\frac{1}{\varepsilon_{1}}\left|\left(u_{n} \vee v_{n}\right)(x)-(u \vee v)(x)\right|\right) \\
\quad \leq & \frac{1}{4}\left[M\left(\frac{4}{\varepsilon_{1}}\left|u_{n}(x)\right|\right)+M\left(\frac{4}{\varepsilon_{1}}\left|v_{n}(x)\right|\right)\right. \\
& \left.+M\left(\frac{4}{\varepsilon_{1}}|u(x)|\right)+M\left(\frac{4}{\varepsilon_{1}}|v(x)|\right)\right] \leq \frac{1}{4}\left[\frac{1}{2} f_{1}\right. \\
& +\frac{1}{2} M\left(\frac{8}{\varepsilon_{1}}|u(x)|\right)+\frac{1}{2} f_{2}+\frac{1}{2} M\left(\frac{8}{\varepsilon_{1}}|v(x)|\right) \\
& \left.+M\left(\frac{4}{\varepsilon_{1}}|u(x)|\right)+M\left(\frac{4}{\varepsilon_{1}}|v(x)|\right)\right],
\end{aligned}
$$

for a.e. $x \in \Omega$.

By Lebesgue's theorem, we get $\int_{\Omega} M\left(\left(1 / \varepsilon_{1}\right) \mid\left(u_{n} \vee v_{n}\right)(x)-\right.$ $(u \vee v)(x) \mid) d x \longrightarrow 0$, as $n \longrightarrow \infty$; this is a contradiction. Consequently, $\left\|u_{n} \vee v_{n}-u \vee v\right\|_{(M)} \longrightarrow 0$, as $n \longrightarrow \infty$. Similarly, 
we can deduce that $\left\|D\left(u_{n} \vee v_{n}\right)-D(u \vee v)\right\|_{(M)} \longrightarrow 0, \| u_{n} \wedge$ $v_{n}-u \wedge v \|_{(M)} \longrightarrow 0$ and $\left\|D\left(u_{n} \wedge v_{n}\right)-D(u \wedge v)\right\|_{(M)} \longrightarrow 0$, as $n \longrightarrow \infty$; that is, the mappings $\vee$ and $\wedge$ are continuous.

A function $u$ is called a (weak) solution of (12) if $u \in$ $W_{0}^{1} L_{M}(\Omega), G(u) \in L_{\bar{P}}(\Omega)$ and $u$ satisfies the following:

$$
\langle A u, v\rangle+\int_{\Omega} G(u)(x) v(x) d x=0,
$$

$$
\text { for all } v \in W_{0}^{1} L_{M}(\Omega) \text {. }
$$

A function $u$ is called a subsolution (resp. supersolution) of (12) if $u \in W_{0}^{1} L_{M}(\Omega), G(u) \in L_{\bar{P}}(\Omega)$, and (16) holds with "=" replaced with " $\leq$ " (resp. " $\geq$ ") for every nonnegative functions $v$ in $W_{0}^{1} L_{\mathrm{M}}(\Omega)$.

By Young inequality and $M \in \Delta_{2}$, there exist $K_{1}>1$ and $\tilde{u}_{1}>0$, such that $\bar{M}(\phi(u))+M(u)=u \phi(u) \leq M(2 u) \leq$ $K_{1} M(u)+M\left(2 \widetilde{u}_{1}\right)$ for all $u>0$. Hence,

$$
\bar{M}(\phi(u)) \leq\left(K_{1}-1\right) M(u)+M\left(2 \widetilde{u}_{1}\right) .
$$

Theorem 6. Let $\underline{u}$ and $\bar{u}$ be a subsolution and a supersolution of problem (12), respectively, such that $\underline{u} \leq \bar{u}$. Assume (H1)-(H3) and the following local growth condition for the nonlinearity g:

$$
|g(x, s, \xi)| \leq k_{1}(x)+c \bar{P}^{-1}(M(|\xi|))
$$

for a.e. $x \in \Omega$, all $\xi \in \mathbb{R}^{N}$, and all $s \in[\underline{u}(x), \bar{u}(x)]$, with $k_{1} \in E_{\bar{P}}(\Omega), k_{1} \geq 0, c \geq 0$. Then there exists at least one solution $u \in W_{0}^{1} L_{M}(\Omega)$ of problem (12) with $u \in[\underline{u}, \bar{u}]:=\{v \in$ $\left.W_{0}^{1} L_{M}(\Omega): \underline{u} \leq v \leq \bar{u}\right\}$.

Proof. Denote $W_{0}^{1} L_{M}(\Omega)=V$. For $x \in \Omega, u \in V$, we put

$$
T u(x)= \begin{cases}\bar{u}(x), & \text { if } u(x)>\bar{u}(x) \\ u(x), & \text { if } \underline{u}(x) \leq u(x) \leq \bar{u}(x) \\ \underline{u}(x), & \text { if } u(x)<\underline{u}(x)\end{cases}
$$

for $u \in V$.

Then $T u=u \vee u+u \wedge \bar{u}-u$. By Lemma $5, T: V \longrightarrow V$ is continuous. It is easy to see that $T$ is bounded.

We define the cutoff function $f: \Omega \times \mathbb{R} \rightarrow \mathbb{R}$ given by

$$
f(x, s)= \begin{cases}\phi(s-\bar{u}(x)), & \text { if } s>\bar{u}(x) \\ 0, & \text { if } \underline{u}(x) \leq s \leq \bar{u}(x) \\ -\phi(\underline{u}(x)-s), & \text { if } s<\underline{u}(x),\end{cases}
$$

for $x \in \Omega, s \in \mathbb{R}$. Then $f$ satisfies the following condition:

$$
|f(x, s)| \leq \phi(|\underline{u}|+|\bar{u}|+|s|),
$$

for $x \in \Omega$ and all $s \in \mathbb{R}$.

Since $M$ is convex and $M \in \Delta_{2}$, there exist $K_{2}>1$ and $\widetilde{u}_{2}>0$ such that $M(|u|) \leq\left(K_{2} / 2\right)[M(u-\bar{u}(x))+M(|\bar{u}(x)|)]+$ $M\left(2 \widetilde{u}_{2}\right)$ whenever $u>\bar{u}(x)$, and $M(|u|) \leq\left(K_{2} / 2\right)[M(\underline{u}(x)-$
$u)+M(|\underline{u}(x)|)]+M\left(2 \widetilde{u}_{2}\right)$ whenever $u<\underline{u}(x)$ for $x \in \Omega$, $u \in \mathbb{R}$. For all $u \in V$, we have

$$
\begin{aligned}
& \int_{\Omega} f(x, u(x)) u(x) d x \\
& =\int_{\{u>\bar{u}\}} \phi(u(x)-\bar{u}(x))(u(x)-\bar{u}(x)) d x \\
& +\int_{\{u>\bar{u}\}} \phi(u(x)-\bar{u}(x)) \bar{u}(x) d x \\
& +\int_{\{u<\underline{u}\}} \phi(\underline{u}(x)-u(x))(\underline{u}(x)-u(x)) d x \\
& -\int_{\{u<\underline{u}\}} \phi(\underline{u}(x)-u(x)) \underline{u}(x) d x \\
& \geq \int_{\{u>\bar{u}\}} M(u(x)-\bar{u}(x)) d x \\
& -\int_{\{u>\bar{u}\}} \bar{M}\left(\varepsilon_{1} \phi(u(x)-\bar{u}(x))\right) d x \\
& -\int_{\{u>\bar{u}\}} M\left(\frac{1}{\varepsilon_{1}} \bar{u}(x)\right) d x \\
& +\int_{\{u<\underline{u}\}} M(\underline{u}(x)-u(x)) d x \\
& -\int_{\{u<\underline{u}\}} \bar{M}\left(\varepsilon_{1} \phi(\underline{u}(x)-u(x))\right) d x \\
& -\int_{\{u<\underline{u}\}} M\left(\frac{1}{\varepsilon_{1}} \underline{u}(x)\right) d x \\
& \geq\left[1-\varepsilon_{1}\left(K_{1}-1\right)\right] \int_{\{u>\bar{u}\}} M(u(x)-\bar{u}(x)) d x \\
& +\left[1-\varepsilon_{1}\left(K_{1}-1\right)\right] \int_{\{u<\underline{u}\}} M(\underline{u}(x)-u(x)) d x \\
& -C_{1}
\end{aligned}
$$

$$
\begin{aligned}
& \geq\left[1-\varepsilon_{1}\left(K_{1}-1\right)\right] \frac{2}{K_{2}} \int_{\Omega} M(|u(x)|) d x-C_{2} \\
& =\frac{1}{K_{2}} \int_{\Omega} M(|u(x)|) d x-C_{2},
\end{aligned}
$$

where $\varepsilon_{1}=1 / 2\left(K_{1}-1\right)$ and the constants $C_{1}, C_{2}>0$.

Define $\Gamma_{T}: V \longrightarrow V^{*}$,

$$
\begin{aligned}
\left\langle\Gamma_{T} u, w\right\rangle:= & \int_{\Omega} \sum_{i=1}^{N} a_{i}(x, T u(x), D u(x)) \frac{\partial w(x)}{\partial x_{i}} d x \\
& +\lambda \int_{\Omega} f(x, u(x)) w(x) d x \\
& +\int_{\Omega} G(T u)(x) w(x) d x,
\end{aligned}
$$

$\forall w \in V$, where $\lambda>0$ is a parameter to be specified later. Then $\Gamma_{T}$ is well defined. 
Since $M \in \Delta_{2}$, there exists a sequence $\left\{w_{j}\right\}_{j=1}^{\infty} \subset \mathscr{D}(\Omega)$ such that $\left\{w_{j}\right\}_{j=1}^{\infty}$ dense in $V$. Let $V_{m}=\operatorname{span}\left\{w_{1}, \ldots, w_{m}\right\}$ and consider $\left.\Gamma_{T}\right|_{V_{m}} \cdot \int_{\Omega}|D u| d x$ and $\|D u\|_{(M)}$ are two norms of $V_{m}$ equivalent to the usual norm of finite dimensional vector spaces.

Similar to the proof of Proposition 3.1 in [22], we can deduce that the mapping $\left.u \longrightarrow \Gamma_{T}\right|_{V_{m}} u: V_{m} \longrightarrow V_{m}^{*}$ is continuous.

By (H3), (18), and (22),

$$
\begin{aligned}
& \left\langle\Gamma_{T} u, u\right\rangle \geq v \int_{\Omega} M(|D u(x)|) d x-\int_{\Omega} k(x) d x+\frac{\lambda}{K_{2}} \\
& \cdot \int_{\Omega} M(|u(x)|) d x-\lambda C_{2} \\
& \quad-\left[c \varepsilon_{2} \int_{\Omega} M(|D T u(x)|) d x\right. \\
& \left.\quad+c \int_{\Omega} P\left(\frac{1}{\varepsilon_{2}}|u(x)|\right) d x\right]-C_{0}\|u\|_{1, M} \geq \frac{v}{2} \\
& \cdot \int_{\Omega} M(|D u(x)|) d x+\left(\frac{\lambda}{K_{2}}-1-c K_{3}\right) \\
& \cdot \int_{\Omega} M(|u(x)|) d x-C_{3}-C_{0}\|u\|_{1, M},
\end{aligned}
$$

for every $u \in V$, where $\varepsilon_{2}=v / 2 c, K_{3}>0$ such that $P\left(\left(1 / \varepsilon_{2}\right)|u(x)|\right) \leq K_{3} M(|u(x)|)$ and the constants $C_{3}, C_{0}>0$. Let $\lambda>K_{2}\left(1+c K_{3}\right)$. Then we can deduce that

$$
\left\langle\Gamma_{T} u, u\right\rangle \geq \frac{v}{2} \int_{\Omega} M(|D u(x)|) d x-C_{3}-C_{0}\|u\|_{1, M} .
$$

By Lemma 1, we get

$$
\begin{gathered}
\frac{\int_{\Omega} M(|D u(x)|) d x}{\|u\|_{1, M}} \geq \frac{1}{1+\operatorname{diam} \Omega} \\
\cdot \frac{\int_{\Omega} M(|D u(x)|) d x}{\|D u\|_{(M)}} \geq \frac{C}{2(1+\operatorname{diam} \Omega)} \\
. \frac{\int_{\Omega} \phi(|D u(x) / 2|)(|D u(x)| / 2) d x}{\int_{\Omega}(|D u(x)| / 2) d x}
\end{gathered}
$$

where the constant $C>0$. By Lemma 2 , we immediately have

$$
\frac{\int_{\Omega} M(|D u(x)|) d x}{\|u\|_{1, M}} \longrightarrow+\infty \quad \text { as }\|u\|_{1, M} \longrightarrow+\infty .
$$

Combining (25) and (27), we obtain

$$
\frac{\left\langle\Gamma_{T} u, u\right\rangle}{\|u\|_{1, M}} \longrightarrow+\infty \quad \text { as }\|u\|_{1, M} \longrightarrow+\infty .
$$

By Remark 2.1 in [22], for every $m$, there is a Galerkin solution $u_{m} \in V_{m}$ such that

$$
\left\langle\Gamma_{T} u_{m}, v\right\rangle=0, \quad \forall v \in V_{m} .
$$

By the density of $\left\{w_{m}\right\}$, we get

$$
\left\langle\Gamma_{T} u_{m}, v\right\rangle=0, \quad \forall v \in V .
$$

As the same proof in [22], we can deduce that the sequence $\left\{u_{m}\right\}$ is bounded in $V$ and there exists $u_{0} \in V$ and a subsequence $\left\{u_{k}\right\}$ of $\left\{u_{m}\right\}$, such that

$$
\begin{aligned}
u_{k} \rightarrow u_{0} & \text { weakly in } V \text { for } \sigma\left(\prod L_{M}, \prod E_{\bar{M}}\right), \\
u_{k} \longrightarrow u_{0} & \text { strongly in } L_{M}(\Omega), \\
u_{k} \longrightarrow u_{0} & \text { strongly in } L_{P}(\Omega), \\
u_{k} \longrightarrow u_{0} & \text { a.e. in } \Omega, \\
\Gamma_{T} u_{k} \rightarrow 0 & \text { weakly in } V^{*} \text { for } \sigma\left(\prod L_{\bar{M}}, \prod E_{M}\right),
\end{aligned}
$$

as $k \longrightarrow+\infty$.

From (21), $\left\{f\left(x, u_{k}(x)\right)\right\}$ is bounded in $L_{\bar{M}}(\Omega)$. By Lemma 4.4 of [17],

$$
\begin{aligned}
& f\left(x, u_{k}(x)\right) \rightarrow f\left(x, u_{0}(x)\right) \\
& \text { weakly in } L_{\bar{M}}(\Omega) \text { for } \sigma\left(L_{\bar{M}}(\Omega), E_{M}(\Omega)\right),
\end{aligned}
$$

as $k \longrightarrow+\infty$.

On the other hand, thanks to (32) and (33), we have

$$
\begin{gathered}
\int_{\Omega} f\left(x, u_{k}(x)\right)\left(u_{k}(x)-u_{0}(x)\right) d x \longrightarrow 0, \\
\int_{\Omega} g\left(x, T u_{k}(x), D T u_{k}(x)\right)\left(u_{k}(x)-u_{0}(x)\right) d x \longrightarrow 0,
\end{gathered}
$$

as $k \longrightarrow+\infty$. Thus we obtain that

$$
\begin{gathered}
\int_{\Omega} \sum_{i=1}^{N} a_{i}\left(x, T u_{k}(x), D u_{k}(x)\right) \\
\cdot\left(\frac{\partial u_{k}(x)}{\partial x_{i}}-\frac{\partial u_{0}(x)}{\partial x_{i}}\right) d x \rightarrow 0,
\end{gathered}
$$

as $k \longrightarrow+\infty$.

Similar to the proof of Proposition 3.1 in [22], we can construct a subsequence still denoted by $\left\{u_{k}\right\}$ such that

$$
D u_{k} \longrightarrow D u_{0} \quad \text { a.e. in } \Omega \text {, as } k \longrightarrow+\infty \text {. }
$$

Hence,

$$
\sum_{i=1}^{N} a_{i}\left(x, T u_{k}, D u_{k}\right) \longrightarrow \sum_{i=1}^{N} a_{i}\left(x, T u_{0}, D u_{0}\right)
$$

a.e. in $\Omega$,

as $k \longrightarrow+\infty$.

Following the lines of Theorem 1 in [2], we can deduce that $\underline{u} \leq u_{k} \leq \bar{u}$ for every $k \in \mathbb{N}$. By (34), $\underline{u} \leq u_{0} \leq \bar{u}$.

Denote $\Omega_{0}=\left\{x \in \Omega: \underline{u}(x) \leq u_{0}(x) \leq \bar{u}(x)\right\}$ and $\Omega_{k}=$ $\left\{x \in \Omega: \underline{u}(x) \leq u_{k}(x) \leq \bar{u}(x)\right\}$. Then meas $\Omega \backslash \Omega_{k}=$ meas $\Omega \backslash$ 
$\Omega_{0}=0$, for every $k \in \mathbb{N}$. It follows from (39) that, passing to a subsequence if necessary,

$$
D T u_{k} \longrightarrow D T u_{0} \quad \text { a.e. in } \Omega \text {, as } k \longrightarrow+\infty \text {. }
$$

Therefore,

$$
\begin{array}{r}
g\left(x, T u_{k}, D T u_{k}\right) \longrightarrow g\left(x, T u_{0}, D T u_{0}\right) \\
\text { a.e. in } \Omega, \text { as } k \longrightarrow+\infty .
\end{array}
$$

Since $\left\{u_{k}\right\}$ and $\left\{T u_{k}\right\}$ are bounded in $V,\left\{\sum_{i=1}^{N} a_{i}\left(x, T u_{k}, D u_{k}\right)\right\}$ is bounded in $L_{\bar{M}}(\Omega)$ and $\left\{g\left(x, T u_{k}, D T u_{k}\right)\right\}$ is bounded in $L_{\bar{p}}(\Omega)$. By Lemma 4.4 of [17], $\Gamma_{T} u_{k} \rightarrow \Gamma_{T} u_{0}$ weakly in $V^{*}$ for $\sigma\left(\prod L_{\bar{M}}, \prod E_{M}\right)$. Thanks to (35), one has $\left(\Gamma_{T} u_{0}, v\right)=0$, for any $v \in V$. Therefore, we obtain that $u_{0}$ is a solution of (12).

Under the assumptions of Theorem 6, we define

$$
\begin{aligned}
\mathcal{S} & =\{u \\
& \in W_{0}^{1} L_{M}(\Omega): u \text { is a solution of }(12) \text { and } \underline{u} \leq u \\
& \leq \bar{u}\} .
\end{aligned}
$$

Theorem 7. Under the assumptions of Theorem 6, the set $\mathcal{S}$ is compact in $W_{0}^{1} L_{M}(\Omega)$.

Proof. Let $\left\{u_{n}\right\}$ be a sequence in $\mathcal{S}$. It follows from the coerciveness of $\Gamma_{T}$ that $\left\{u_{n}\right\}$ is bounded in $W_{0}^{1} L_{M}(\Omega)$. As the same proof of Theorem 6 , there exists that $u_{0}$ is a solution of (12) and $\underline{u} \leq u_{0} \leq \bar{u}$, i.e., $u_{0} \in \mathcal{S}$.

To show that the set $\mathcal{S}$ is directed with respect to the usual pointwise order, the following additional assumption on the coefficients $a_{i}: \Omega \times \mathbb{R} \times \mathbb{R}^{N} \longrightarrow \mathbb{R}$ is required.

(H4) Let a nonnegative function $k \in L_{\bar{M}}(\Omega)$ and a continuous function $\omega: \mathbb{R}^{+} \longrightarrow \mathbb{R}^{+}$exist such that

$$
\begin{gathered}
\left|a_{i}(x, s, \xi)-a_{i}\left(x, s^{\prime}, \xi\right)\right| \leq\left[k(x)+\bar{P}^{-1}(M(|s|))\right. \\
\left.+\bar{P}^{-1}\left(M\left(\left|s^{\prime}\right|\right)\right)+\bar{M}^{-1}(M(|\xi|))\right] \omega\left(\left|s-s^{\prime}\right|\right)
\end{gathered}
$$

holds for a.e. $x \in \Omega$, for all $s, s^{\prime} \in \mathbb{R}$ and for all $\xi \in \mathbb{R}^{N}$, where $\omega$ satisfies $\int_{0^{+}}(d r / \omega(r))=+\infty$, that is, for every $\varepsilon>0, \int_{0}^{\varepsilon}(d r / \omega(r))=+\infty$.

Similar to the proof of [1, Theorem 3.20], we can deduce the following result.

Theorem 8. Assume hypotheses (H1)-(H4), and let $u_{1}$ and $u_{2}$ be subsolutions of (12) such that the Nemytskii operator

$$
G:\left[u_{1} \wedge u_{2}, u_{1} \vee u_{2}\right] \longrightarrow L_{\bar{M}}(\Omega)
$$

is well defined. Then $u_{1} \vee u_{2}$ is a subsolution of (12). Analogously, if $u_{1}$ and $u_{2}$ are supersolutions of (12) with the same assumption on the Nemytskii operator $G$, then $u_{1} \wedge u_{2}$ is a supersolution.
Theorem 9. Let the assumptions of Theorem 6 and (H4) hold. Then the following assertions about $\mathcal{S}$ are true.

(a) $\mathcal{S}$ is a direct set in both directions; that is, if $u_{1}, u_{2} \in \mathcal{S}$ then there exist $u, v \in \mathcal{S}$ such that $u \geq u_{1} \vee u_{2}$ and $v \leq u_{1} \wedge u_{2}$.

(b) $\mathcal{S}$ possesses extremal elements; i.e., there are $u_{*}, u^{*} \in \mathcal{S}$ such that $u_{*} \leq u \leq u^{*}$, for all $u \in \mathcal{S}$.

Proof. (a) Let $u_{1}, u_{2} \in \mathcal{S}$. Then $u_{1}$ and $u_{2}$ are both subsolutions and supersolutions of (12). It follows, from Theorem 8, $u_{1} \vee u_{2}$ is a subsolution and $u_{1} \wedge u_{2}$ is a supersolution of (12). The claim in (a) is now a straightforward consequence of Theorem 6.

(b) Since $W_{0}^{1} L_{M}(\Omega)$ is separable, there exists a countable, dense subset $\left\{w_{n}: n \in \mathbb{N}\right\}$ of $\mathcal{S}$. Let $u_{1}=w_{1}$. By (a), we can select $u_{n+1} \in \mathcal{S}$ such that $u_{n} \vee w_{n} \leq u_{n+1} \leq \bar{u}$. Thus, we get a bounded increasing sequence $\left\{u_{n}\right\} \subset \mathcal{S}$. Consequently, $\lim _{n \rightarrow \infty} u_{n}(x)=\sup _{n \in \mathbb{N}} u_{n}(x):=u^{*}(x)$, for a.e. $x \in \Omega$, and there exists a subsequence $\left\{u_{k}\right\} \subset\left\{u_{n}\right\}$ such that $u_{k} \rightarrow u^{*}$ weakly in $W_{0}^{1} L_{M}(\Omega)$ for $\sigma\left(\prod L_{M}, \prod E_{\bar{M}}\right)$ as $k \longrightarrow+\infty$. Similar to the proof of Theorem 6 , we can deduce that $u^{*} \in \mathcal{S}$. From the density of $\left\{w_{n}: n \in \mathbb{N}\right\}$, we can get that $u^{*}$ is the greatest element of $\mathcal{S}$. The existence of the smallest element of $\mathcal{S}$ can be deduced in the same way.

Remark 10. A special case in Theorem 6 is that $P=M$. In this case, choice $M(t)=|t|^{p}$ leads to Theorem 3.17 in [1].

Remark 11. The above results can be extended to the more general situation of Musielak-Orlicz-Sobolev spaces following our method developed in this paper.

\section{Data Availability}

No data were used to support this study.

\section{Conflicts of Interest}

The authors declare that there are no conflicts of interest regarding the publication of this paper.

\section{Acknowledgments}

The first author is supported by 'Chen Guang' Project (supported by Shanghai Municipal Education Commission and Shanghai Education Development Foundation) (10CGB25) and the Teaching Reform Project of Jianqiao University (JGXM201608). The second author is supported by the National Natural Science Foundation of China (11371279) and the Fundamental Research Funds for the Central Universities.

\section{References}

[1] S. Carl, V. K. Le, and D. Motreanu, Nonsmooth Variational Problems and their Inequalities: Comparison Principles and Applications, Springer Monographs in Mathematics, Springer, New York, NY, USA, 2007. 
[2] L. F. Faria, O. H. Miyagaki, D. Motreanu, and M. Tanaka, "Existence results for nonlinear elliptic equations with LerayLions operator and dependence on the gradient," Nonlinear Analysis. Theory, Methods \& Applications. An International Multidisciplinary Journal, vol. 96, pp. 154-166, 2014.

[3] M. Mihailescu and V. Radulescu, "Existence and multiplicity of solutions for quasilinear nonhomogeneous problems: an Orlicz-Sobolev space setting," Journal of Mathematical Analysis and Applications, vol. 330, no. 1, pp. 416-432, 2007.

[4] V. K. Le, "On a sub-supersolution method for variational inequalities with Leray-Lions operators in variable exponent spaces," Nonlinear Analysis. Theory, Methods \& Applications. An International Multidisciplinary Journal, vol. 71, no. 7-8, pp. 3305-3321, 2009.

[5] X. L. Fan, "Differential equations of divergence form in Musielak-Sobolev spaces and a sub-supersolution method," Journal of Mathematical Analysis and Applications, vol. 386, no. 2, pp. 593-604, 2012.

[6] G. Dong and Z. Shi, "An existence theorem for weak solutions for a class of elliptic partial differential systems in Orlicz spaces," Nonlinear Analysis: Theory, Methods \& Applications, vol. 68, no. 4, pp. 1037-1042, 2008.

[7] G. Dong, "An existence theorem for weak solutions for a class of elliptic partial differential systems in general Orlicz-Sobolev spaces," Nonlinear Analysis: Theory, Methods \& Applications, vol. 69, no. 7, pp. 2049-2057, 2008.

[8] G. Dong, "Elliptic equations with measure data in Orlicz spaces," Electronic Journal of Differential Equations, vol. 76, pp. $1-10,2008$.

[9] G. Dong and X. Fang, "Variational Inequalities with Multivalued Lower Order Terms and Convex Functionals in OrliczSobolev Spaces," Journal of Function Spaces, vol. 2015, Article ID 321437, 10 pages, 2015.

[10] G. Dong and X. Fang, "Differential equations of divergence form in separable Musielak-Orlicz-Sobolev spaces," Boundary Value Problems, Paper No. 106, 19 pages, 2016.

[11] V. K. Le, "On variational and quasi-variational inequalities with multivalued lower order terms and convex functionals," Nonlinear Analysis: Theory, Methods \& Applications, vol. 94, pp. 12-31, 2014

[12] S. Pollock and Y. Zhu, "Discrete comparison principles for quasilinear elliptic PDE," https://arxiv.org/abs/1708.02301.

[13] N. S. Papageorgiou, C. Vetro, and F. Vetro, "Extremal solutions and strong relaxation for nonlinear multivalued systems with maximal monotone terms," Journal of Mathematical Analysis and Applications, vol. 461, no. 1, pp. 401-421, 2018.

[14] R. A. Adams and J. J. Fournier, Sobolev Spaces, vol. 140, Academic Press, New York, NY, USA, 2nd edition, 2003.

[15] M. A. Krasnosel'skil and J. B. Rutickil, Convex functions and Orlicz spaces, P. Noordhoff Ltd., Groningen, Netherlands, 1961.

[16] S. Chen, Geometry of Orlicz Spaces, Dissertationes Mathematicae, Warszawa, Pland, 1996.

[17] J.-P. Gossez, "Nonlinear elliptic boundary value problems for equations with rapidly (or slowly) increasing coefficients," Transactions of the American Mathematical Society, vol. 190, pp. 163-205, 1974.

[18] G. M. Lieberman, "The natural generalization of the natural conditions of Ladyzhenskaya an Ural'tseva for elliptic equations," Communications in Partial Differential Equations, vol. 16, no. 2-3, pp. 311-361, 1991.
[19] R. Landes, "Quasilinear elliptic operators and weak solutions of the Euler equation," Manuscripta Mathematica, vol. 27, no. 1, pp. $47-72,1979$.

[20] J. F. Rodrigues and R. Teymurazyan, "On the two obstacles problem in Orlicz-Sobolev spaces and applications," Complex Variables and Elliptic Equations, vol. 56, no. 7-9, pp. 769-787, 2011.

[21] M. García-Huidobro, V. K. Le, R. Manasevich, and K. Schmitt, "On principal eigenvalues for quasilinear elliptic differential operators: an Orlicz-Sobolev space setting," Nonlinear Differential Equations and Applications NoDEA, vol. 6, no. 2, pp. 207225, 1999.

[22] G. Dong and X. Fang, "Existence results for some nonlinear elliptic equations with measure data in Orlicz-Sobolev spaces," Boundary Value Problems, 22 pages, 2015. 


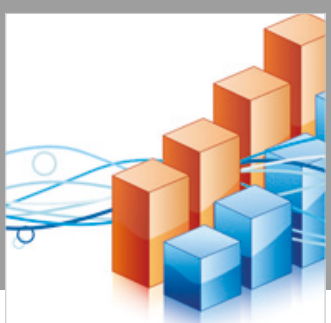

Advances in

Operations Research

\section{-n-m}
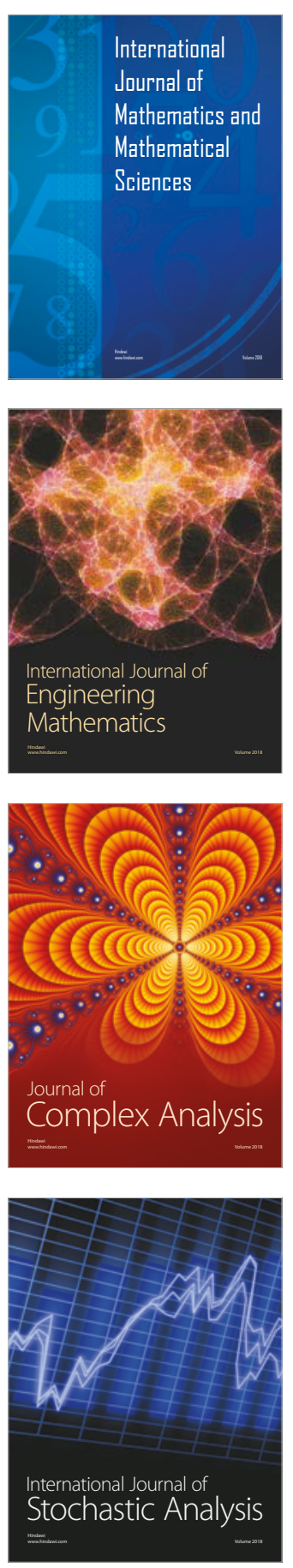
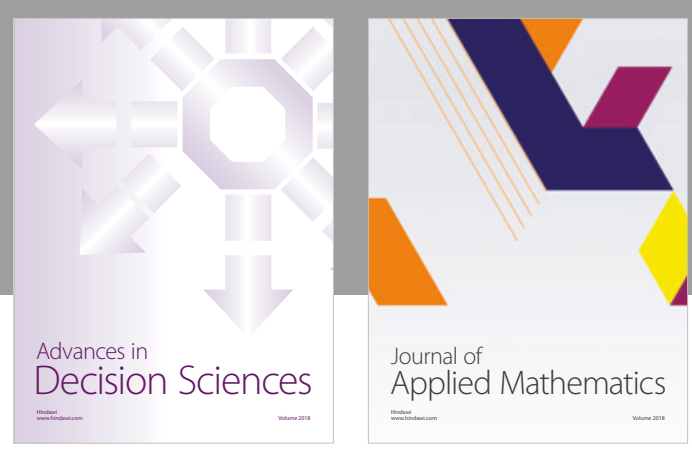

Journal of

Applied Mathematics
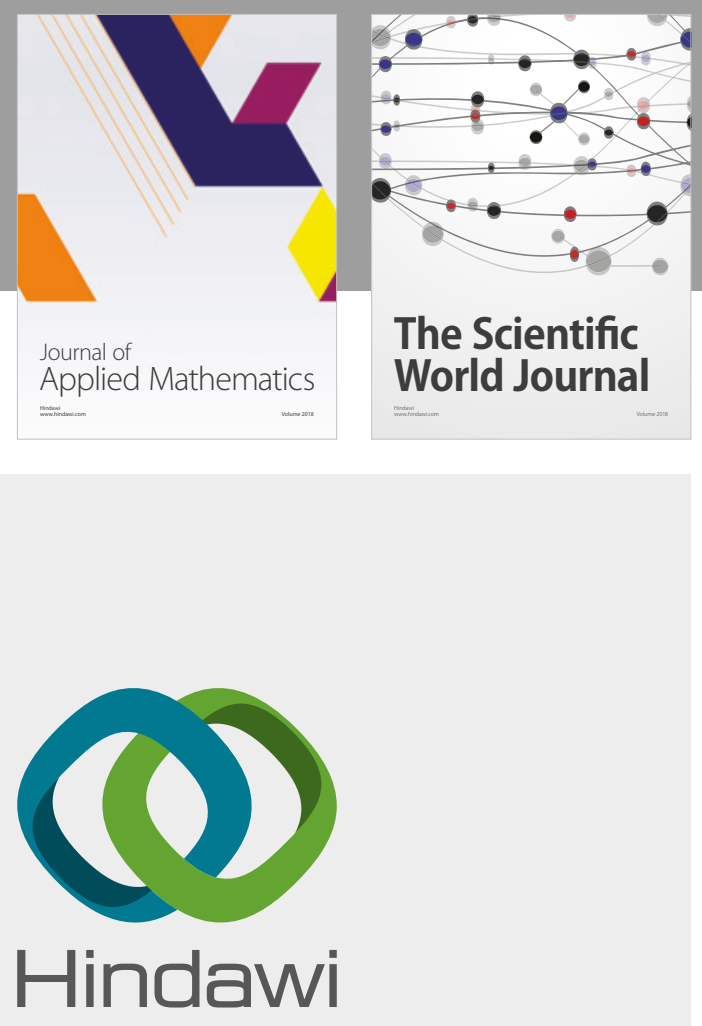

Submit your manuscripts at

www.hindawi.com

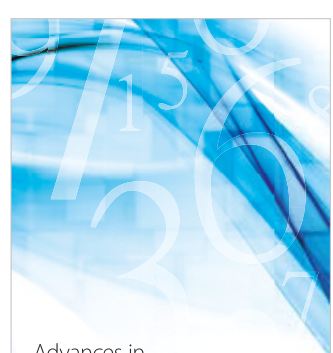

Advances in
Numerical Analysis
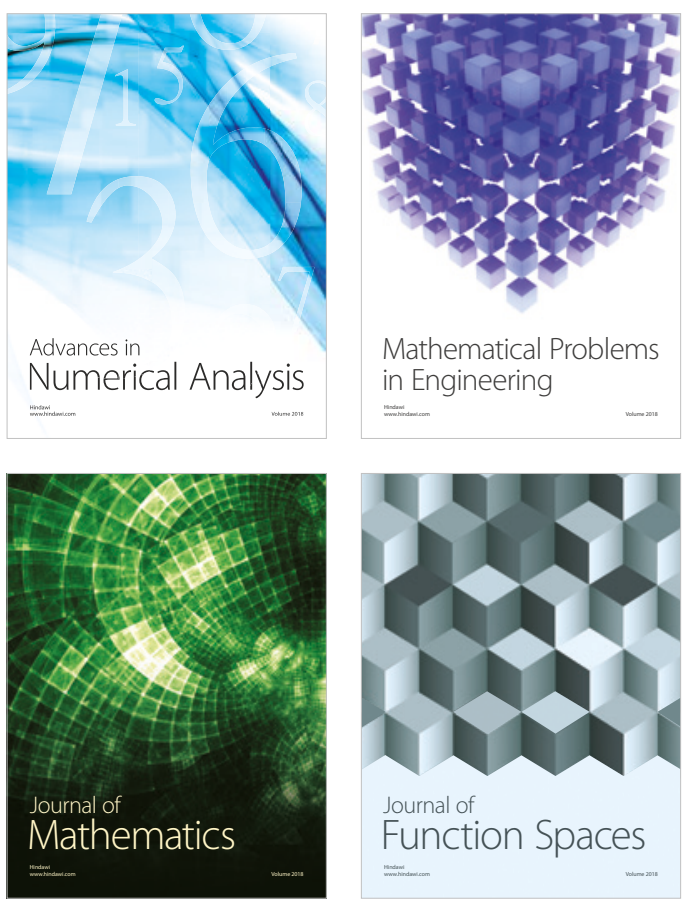

Mathematical Problems in Engineering

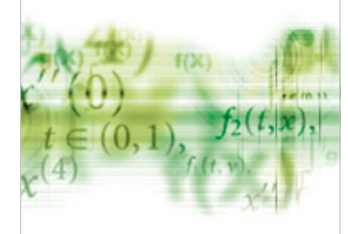

International Journal of

Differential Equations

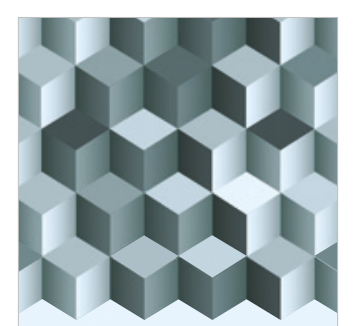

Journal of

Function Spaces

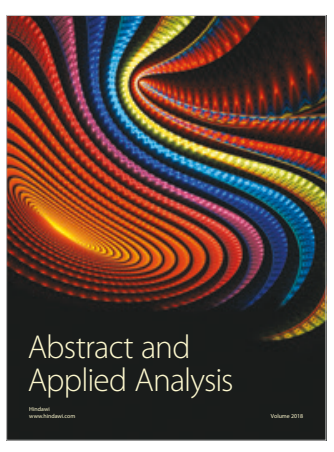

The Scientific

World Journal

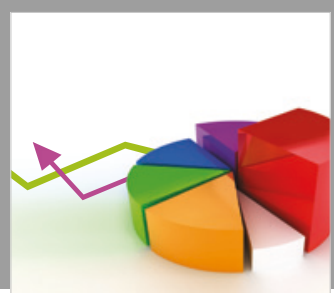

Journal of

Probability and Statistics
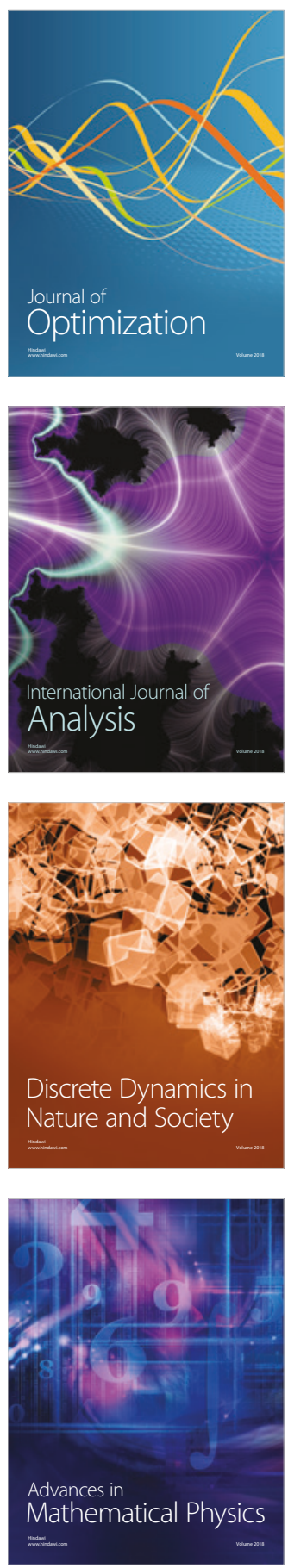